\title{
Optimizing the Use of Current Treatments and Emerging Therapeutic Approaches to Achieve Therapeutic Success in Patients with Inflammatory Bowel Disease
}

\author{
Hiroshi Nakase \\ Department of Gastroenterology and Hepatology, Sapporo Medical University School of Medicine, Sapporo, Japan
}

The current goal of inflammatory bowel disease (IBD) treatment is a symptom-free everyday life accompanied by mucosal healing with minimal use of corticosteroids. Recent therapeutic advances, particularly, the emergence of antitumor necrosis factor (anti-TNF) antibodies, have changed the natural history of IBD. Additionally, these advances also led to the emergence of the therapeutic concept of the "treat to target" strategy. With the development of new drugs and clinical trials, not only biologics but also small molecules have been applied to clinical practice to better individualize and optimize therapy. However, if newer drugs, including antiTNF therapies, are recommended for all patients diagnosed with IBD, a significant number of patients will be overtreated. The basic goal of IBD treatment is still to make the best use of conventional treatments based on IBD pathophysiology. Thus, physicians should be familiar with the modes of action of the available drugs. In this review, the author discusses the existing data for many approved drugs and provide insights for optimizing current treatments for the management of patients with IBD in the era of biologics. (Gut Liver 2020;14:7-19)

Key Words: Mesalamine; Prednisolone; Immunomodulators; Biologics; Janus kinase inhibitors

\section{INTRODUCTION}

In the past two decades, treatment with tumor necrosis factor (TNF)- $\alpha$ inhibitors has achieved better clinical better outcomes in inflammatory bowel disease (IBD) patients. However, not all patients respond to induction therapy with these agents, and of those who initially respond, up to $40 \%$ ultimately lose response due to suboptimal drug exposure, side effects, or other poorly characterized mechanisms. Recently, additional therapies, such as an antibody that blocks the common p40 subunit of interleukin (IL)-12 and IL-23, and an integrin blocker that prevents $\mathrm{T}$ cell trafficking to the gut, were introduced to the market. In addition, other agents including novel anti-trafficking therapies (e.g., sphingosine-1-phosphate receptor modulators), antibodies against p19 (unique to IL-23), and small molecules, such as Janus kinase (JAK) inhibitors are being developing for use in IBD treatment. Furthermore, the management of IBD has evolved from targeting the control of symptoms to targeting the suppression of mucosal inflammation, leading to the concept of "treat to target."

This shift in thinking has been accompanied by the early use of highly effective therapies in patients with a poor prognosis, the acceleration of treatment escalation and the adoption of therapeutic drug monitoring. However, physicians remember that the basic goal of IBD therapy is to make the best use of conventional treatments based on their mechanisms of action (Figs 1 and 2). ${ }^{1}$ In this review, we focus on the significance of conventional therapies in the era of biologics and newly emerging therapies.

\section{CURRENT THERAPEUTIC APPROACHES}

\section{Aminosalicylates}

\section{1) Ulcerative colitis}

There is a consensus among all gastroenterologists concerning the use of aminosalicylates (5-ASA) drugs as the treatment mainstay for mild to moderate ulcerative colitis (UC) because many reports suggested that the long-term use of 5-ASA drugs is safe and useful for maintaining remission. Mesalamine has pleiotropic effects on various signaling pathways, including nuclear factor (NF)- $\mathrm{kB}, \mathrm{Wnt} / \beta$-catenin, peroxisome proliferator-

\footnotetext{
Correspondence to: Hiroshi Nakase

Department of Gastroenterology and Hepatology, Sapporo Medical University School of Medicine, S-1, W-16, Chuo-ku, Sapporo 060-8543, Japan

Tel: +81-11-611-2111, Fax: +81-11-613-1241, E-mail: hiropynakase@gmail.com

Received on May 8, 2018. Revised on October 6, 2018. Accepted on October 12, 2018. Published online March $12,2019$. pISSN 1976-2283 eISSN 2005-1212 https://doi.org/10.5009/gnl18203

() This is an Open Access article distributed under the terms of the Creative Commons Attribution Non-Commercial License (http://creativecommons.org/licenses/by-nc/4.0) which permits unrestricted non-commercial use, distribution, and reproduction in any medium, provided the original work is properly cited.
} 
activated receptor (PPAR)- $\gamma$, mitogen-activated protein kinase (MAPK), and PI3K/Akt, and these effects are thought to be responsible for the inhibition of colonic inflammation (Fig. 3). ${ }^{2,3}$ Recently, Oh-Oka et al. ${ }^{4}$ proposed a novel anti-inflammatory mechanism for mesalamine in colitis that involves the induction of Tregs in the colon via the aryl hydrocarbon receptor pathway, followed by transforming growth factor- $\beta$ activation. Thus, 5-ASAs are mysterious because of their various antiinflammatory properties.

In general, topical mesalazine (a 5-ASA) was able to induce remission of active proctitis and distal colitis in 31\%-80\% (median, $67 \%$ ) of patients compared to $7 \%-11 \%$ of patients treated with placebo in a meta-analysis evaluating 11 trials with a total of 778 patients. ${ }^{5}$ Multinational survey data regarding the initial treatment of mild to moderate UC from the 2nd Asian Organization for Crohn's and Colitis meeting demonstrated that topical 5-ASAs were the most preferred treatment for ulcerative proctitis, followed by oral 5-ASAs. ${ }^{6}$ Combined oral and topical mesalazine therapy appeared to be superior to oral 5-ASAs for the induction of remission of mildly to moderately active UC. A meta-analysis of mesalazine showed a dose-response for clinical improvement for doses of $2.0 \mathrm{~g}, 2.0-2.9 \mathrm{~g}$ and $3.0 \mathrm{~g}$ administered daily $(\mathrm{p}=0.002)^{7}$ A clinical trial demonstrated that compared with $\mathrm{pH}$-dependent-release mesalazine at a dose of 3.6 g/day, multimatrix (MMX)-mesalazine $4.8 \mathrm{~g} /$ day has higher efficacy and shows no difference in safety in patients with mildly to moderately active UC; thus, MMX-mesalazine may be a promising option for inducing remission. ${ }^{8}$

Maintenance of remission might be achieved using mesalazine at lower dosages than are used for induction treatment. ${ }^{9}$ Pancolitis should be treated following the same rules used for left-sided UC; however, systemic steroids should be used sooner

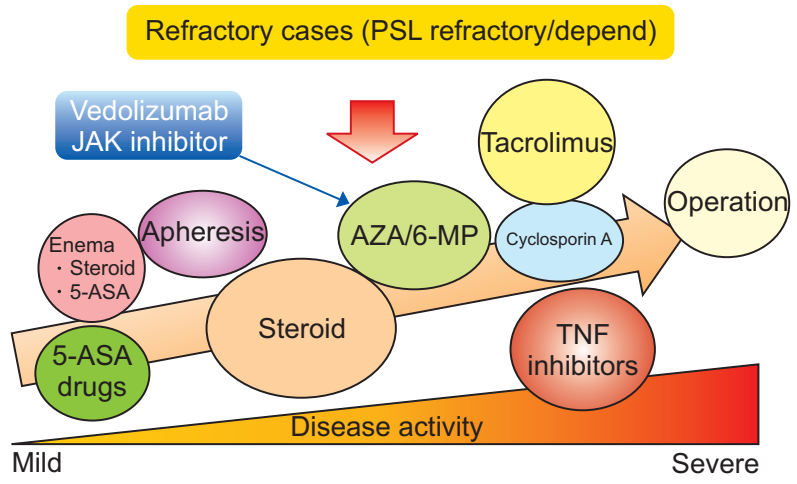

Fig. 1. Medical treatment approaches for ulcerative colitis. PSL, prednisolone; JAK, Janus kinase; AZA, azathioprine; 6-MP, 6-mercaptopurine; 5-ASA, aminosalicylates; TNF, tumor necrosis factor. Modified from Nakase H. How to select and use IBD drugs. Tokyo: Yodosha Co., Ltd., 2015, with permission. ${ }^{1}$

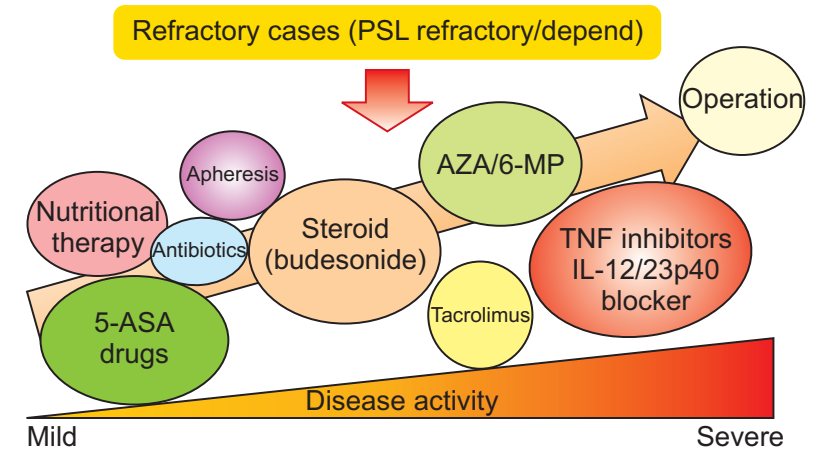

Fig. 2. Medical treatment approaches for Crohn's disease.

PSL, prednisolone; AZA, azathioprine; 6-MP, 6-mercaptopurine; 5-ASA, aminosalicylates; TNF, tumor necrosis factor; IL, interleukin. Modified from Nakase H. How to select and use IBD drugs. Tokyo: Yodosha Co., Ltd., 2015, with permission. ${ }^{1}$

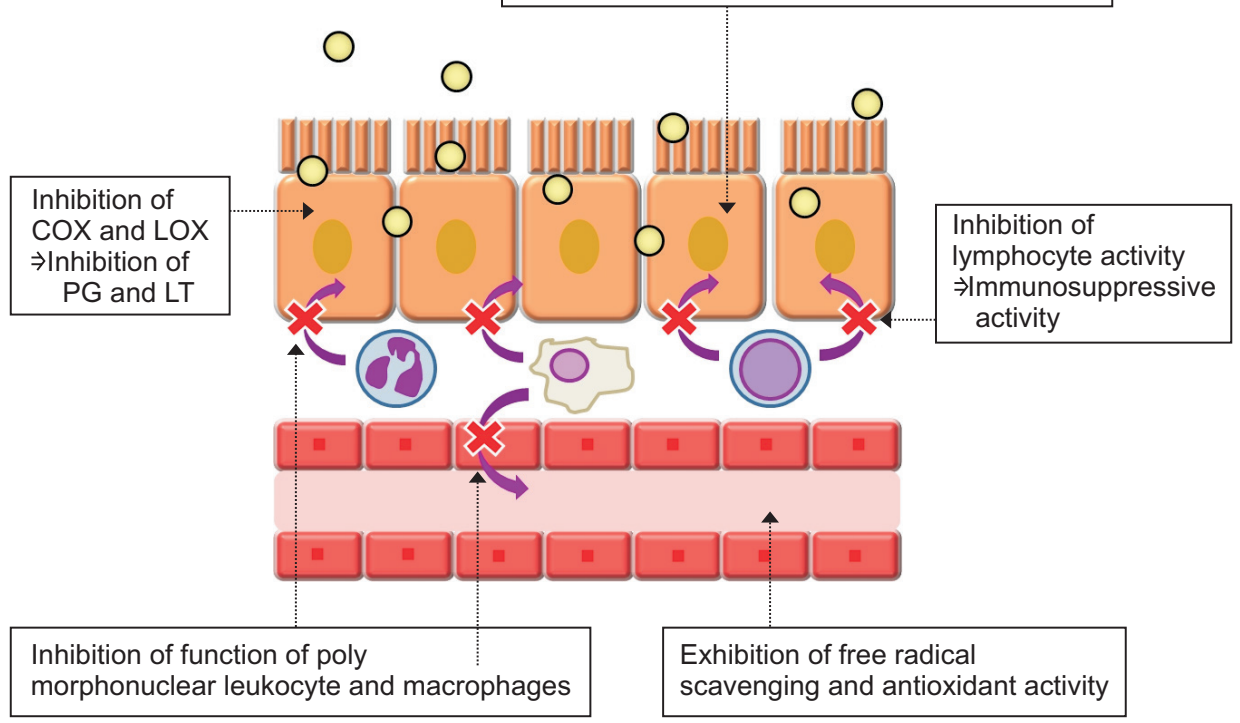

Fig. 3. Mechanism of action of aminosalicylates (5-ASAs) in inflammatory bowel disease.

PPAR $\gamma$, peroxisome proliferatoractivated receptor $\gamma$; NF-kB, nuclear factor-kB; TLR, Toll-like receptor; COX, cyclooxygenase; LOX, lipoxygenase; PG, prostaglandin; LT, leukotriene. Modified from Nakase H. To master the optimal therapy for ulcerative colitis. Tokyo: Igakutokangosha Co., Ltd., 2018, with permission. $^{2}$ 
in patients with pancolitis than in patients with left-sided colitis, depending on the severity. A recent topic of study is patient adherence to oral mesalazine. Generally, adherence to mesalazine among UC patients is poor because of inconvenient dosing regimens. Therefore, reduction of the number of doses is a better way to accommodate patient preferences when prescribing mesalazine. Flourié et al. ${ }^{10}$ performed a multicenter controlled, randomized, investigator-blinded, comparative study designed to investigate the noninferiority of once daily (OD) versus twice daily (BD) administration. This study demonstrated that the primary endpoint, noninferiority in clinical and endoscopic remission with OD versus BD mesalazine at 8 weeks, was met (intent-to-treat population: $52.1 \%$ vs $41.8 \%$, respectively; $95 \%$ confidence interval [Cl], -3.4 to $24.1 ; \mathrm{p}=0.14)$. Improvement of the UC-DAI score (92\% vs 79\%; $\mathrm{p}=0.01$ ) and mucosal healing (87.5\% vs $71.1 \%$; $p=0.007$ ) was significantly better, and the time to remission was significantly shorter with OD dosing. Kruis et al. ${ }^{11}$ suggested that OD dosing could lead to a higher peak concentration and a higher mucosal concentration of mesalazine than divided dosing given at a similar total daily dose. We investigated the mucosal concentration in patients with quiescent UC who were treated with 5-ASA. As expected, the mucosal concentration was higher in quiescent UC patients treated with the $\mathrm{OD}$ regimen than in those treated $\mathrm{BD}$ or three times daily (unpublished data). Our data strongly support both proposal by Kruis et al. and MOTUS study. Therefore, maintaining adherence in patients receiving 5-ASAs is a clinically important issue for preventing UC relapse.

\section{2) Crohn's disease}

Historically, mesalamine and sulfasalazine were preferred as induction agents for mild to moderately active Crohn's disease (CD), despite previous meta-analyses reporting a lack of effectiveness. Guidelines from the American College of Gastroenterology suggest that mesalamine is minimally effective for the treatment of mild to moderate $\mathrm{CD} .{ }^{12}$ A recent network metaanalysis showed that high-dose mesalamine (odds ratio [OR], 2.29; 95\% CI, 1.58 to 3.33) was superior to placebo, although these data conflicted with previous meta-analyses and current clinical practice guidelines. ${ }^{13}$

\section{Corticosteroids}

Corticosteroids (CSs) act via the inhibition of several inflammatory pathways, such as the suppression of interleukin transcription, the induction of $\mathrm{I}_{\kappa} \mathrm{B}$, which stabilizes the $\mathrm{NF}-\kappa \mathrm{B}$ complex, the suppression of arachidonic acid metabolism and the stimulation of lymphocyte apoptosis within the lamina propria of the gut (Fig. 4). ${ }^{14}$ CSs are optimal drugs for controlling the severe intestinal inflammation of IBD. However, long-term use of CSs should be avoided. Experimental data suggested that intestinal epithelial cell-specific deletion of inhibitor of kappa $B$ kinase (IKK) $\beta$ or pharmacological inhibition of $N F-\kappa B$ resulted in exacerbation of acute colitis induced by dextran sodium sulfate. ${ }^{15}$ Inoue et al. ${ }^{16}$ demonstrated that proteasome inhibition (by MG132) reduced T cell-mediated intestinal inflammation, but interrupted both epithelial regeneration and the barrier function of colonic mucosa. These data suggest that long-term use of CSs (high cumulative dose) could affect mucosal regeneration in patients with IBD.

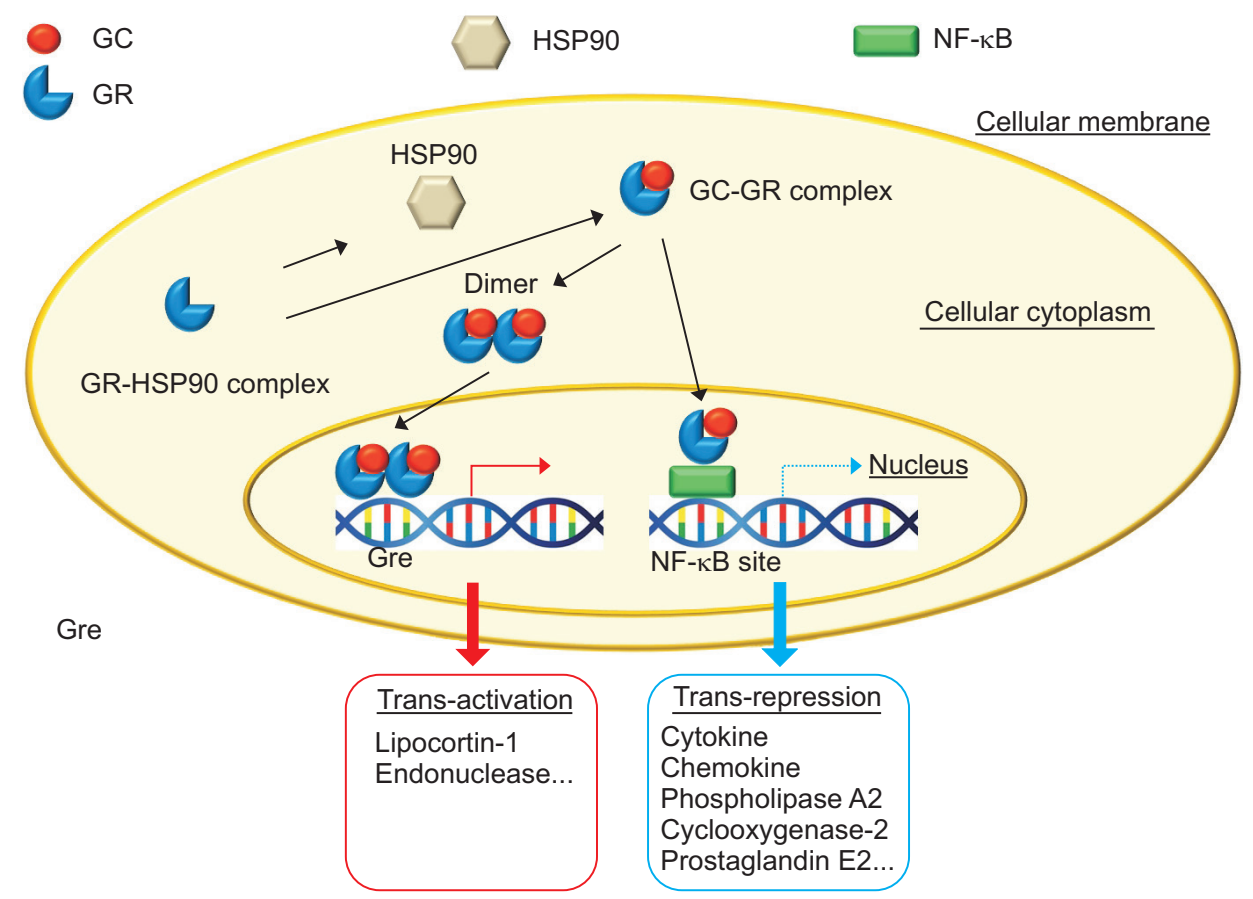

Fig. 4. Mechanism of action of corticosteroids.

GC, glucocorticoid; HSP90, heat shock protein 90; NF-kB, nuclear factor-kB; GR, glucocorticoid receptor; Gre, glucocorticoid response element. Modified from Nakase $\mathrm{H}$. To master the optimal therapy for ulcerative colitis. Tokyo: Igakutokangosha Co., Ltd., 2018, with permission. ${ }^{2}$ 


\section{1) Ulcerative colitis}

CSs have been used since the 1950s in IBD patients, and they remain one of the most effective treatments for disease flares. ${ }^{17}$ In UC, precise evidence concerning the most effective dose and duration of CS therapy is lacking. Evidence for the benefit of oral CS therapy comes from two early studies of active UC. ${ }^{18,19}$ The $60 \mathrm{mg}$ of prednisolone seems to be no more effective than $40 \mathrm{mg}$, but the higher dose is associated with a higher frequency of adverse events. ${ }^{20}$ Current guidelines suggest starting with an initial oral dose of $40-60 \mathrm{mg}(0.75-1 \mathrm{mg} / \mathrm{kg})$ prednisolone daily followed by tapering. ${ }^{21,22}$ Generally, an appropriate regimen for patients with moderately active UC is prednisolone $40 \mathrm{mg} /$ day for 1 week, with subsequent reduction of the dose by $5 \mathrm{mg} /$ day every week, resulting in an 8-week course. It should be noted that a shorter course of $<3$ weeks is associated with early relapse and doses of prednisolone $\leq 15 \mathrm{mg}$ day are ineffective for active disease. In addition, the initial dose and the rate of steroid tapering were associated with neither long-term remission rates nor the duration of remission.

In cases of severe UC, the patient should be admitted to the hospital for intensive treatment under the care of a multidisciplinary team, including a specialist in gastroenterology and a colorectal surgeon. A high dose of CSs is generally given intravenously. A systematic review of 32 trials of steroid therapy for acute severe colitis involving 1,991 patients from 1974 to 2006 reported an overall response to steroids (intravenous hydrocortisone, methylprednisolone, or betamethasone) of $67 \%$ (95\% Cl, 65\% to 69\%). ${ }^{23}$ However, CS treatment should be given for a defined period because extending therapy beyond 7 to 10 days carries no additional benefit. If an adequate response is not achieved during the intravenous administration of CSs, secondline treatment with infliximab, tacrolimus (TAC), or cyclosporine must be considered. Maintenance therapy can be achieved either by continuing infliximab or by administering azathioprine, which replaces TAC or cyclosporine after the acute phase.

\section{2) Crohn's disease}

In active $\mathrm{CD}$ patients, we consider the administration of systemic CSs or budesonide (better candidates have an ileocecal type of $\mathrm{CD}$ ). There is better evidence for the use of budesonide in mild to moderate flares of the ileocecal CD. Coward et al. ${ }^{13}$ reported that $9 \mathrm{mg}$ budesonide was significantly better than 3 or $6 \mathrm{mg}$ budesonide (OR, 2.18; 95\% CI, 1.02 to 4.80), and budesonide was more effective than mesalamine (OR, 1.57; 95\% CI, 1.06 to 2.32 ) and sulfasalazine (OR, 1.91; 95\% CI, 1.04 to 3.61). In addition, CSs were similar to high-dose budesonide (OR, 1.21 ; 95\% CI, 0.84 to 1.76). It has been reported that typical CSassociated side effects are less frequent with budesonide than with systemic CS treatment. Therefore, budesonide $9 \mathrm{mg}$ per day might be the best choice for inducing clinical remission in patients with ileocecal $\mathrm{CD}$. In real clinical practice, $\mathrm{CD}$ patients with extensive intestinal lesions would require systemic CSs.
However, the goal of every IBD therapy is the achievement of steroid-free remission. We should keep in mind that CSs have no role as maintenance therapy in either UC or CD.

\section{Immunomodulators}

Thiopurine drugs: inactive 6-thiopurine (TP) prodrugs 6-thiopurine (azathioprine [AZA] and 6-thioguanine [6-TG]) are converted into pharmacologically active deoxy-6-thioguanosine phosphate (deoxy-6-TGNP). Deoxy-6-TGNP can be incorporated into the de novo synthesis of DNA as a form of 6-TG (Fig. 5). Recent studies have shown that 6-TGNP derived from 6-TP prodrugs binds to Rac1 to form the 6-TGNP-Rac1 complex. The formation of this complex, in turn, induces immunosuppression by blocking Rac1 activation mediated by the Rho guanine nucleotide exchange factor Vav in T lymphocytes, which and the inactivation of Rac1 suppresses the function and survival of CD4+ cells. $^{24}$

\section{1) Ulcerative colitis}

TPs have been used as maintenance treatment for refractory and chronic active IBD patients. Among TPs, AZA is most commonly used. Most physicians consider the effect of TPs to be favorable for patients with refractory UC, particularly in steroiddependent cases. In UC patients, a meta-analysis that reviewed 30 noncontrolled studies and analyzed seven controlled studies confirmed that TP drugs are more effective than placebo for the prevention of relapse in $\mathrm{UC}^{25}$ Since we are in the biologic era, we must recognize the long-term efficacy of TPs. ${ }^{26}$

\section{2) Crohn's disease}

In CD patients, as described for UC patients, TPs have been effective in facilitating the reduction of steroid doses and maintaining remission after induction therapy with steroids. ${ }^{27,28}$ The

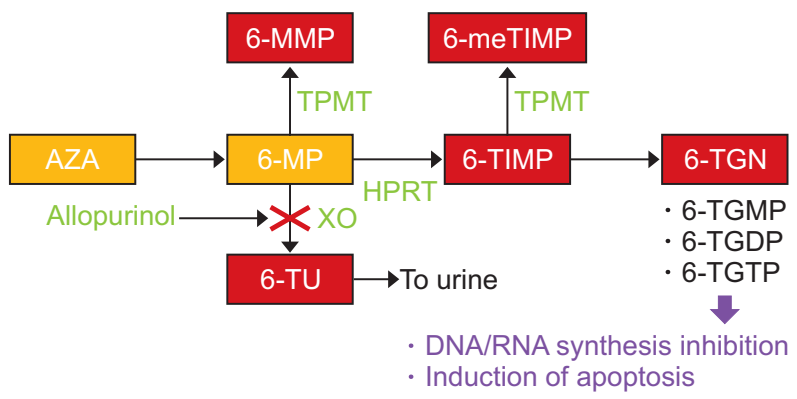

Fig. 5. Metabolic pathway of thiopurines.

6-MMP, 6-methyl-mercaptopurine; 6-meTIMP, 6-methyl-thioinosine monophosphate; TPMT, thiopurine S-methyl transferase; AZA, azathioprine; 6-MP, 6-mercaptopurine; 6-TIMP, 6-thioinosine monophosphate; 6-TGN, 6-thioguanine nucleotides; HPRT, hypoxanthine phosphoribosyl transferase; X0, xanthine oxidase; 6-TU, 6-thiouric acid; 6-TGMP, 6-thioguanosine monophosphate; 6-TGDP, 6-thioguanine diphosphate; 6-TGTP, 6-thioguanosine triphosphate. Modified from Nakase H. To master the optimal therapy for ulcerative colitis. Tokyo: Igakutokangosha Co., Ltd., 2018, with permission. ${ }^{2}$ 
initial induction strategy with steroids may be important, because AZA has a significantly delayed onset of action, with several studies demonstrating clinical efficacy after 2 to 3 months of treatment. ${ }^{29,30}$

\section{3) The difference in TP metabolism between Asian and Western populations}

In Western IBD patients, the appropriate maintenance dose is 2-2.5 mg/day of AZA and 1-1.5 mg/day of 6-mercaptopurine (6-MP). However, in Asian IBD patients, the therapeutic doses of AZA are relatively low (25-100 mg/day) in comparison with those used in the West because of the difference in TP metabolism between Asian and Western populations. A current topic of study in TP metabolism is nucleoside diphosphate-linked to another moiety X hydrolase (NUDT)-15. The physiological function of NUDT15 is considered to be the hydrolysis of 8-oxodGTP generated from reactive oxygen species. ${ }^{31}$ Recent studies indicated that NUDT15 preferentially inactivates TP metabolites over 8-oxo-dGTP, converting molecules such as TGTP and TdGTP to TGMP and TdGMP, respectively. ${ }^{32-34}$ Thus, NUDT15 is a negative regulator that decreases the levels of bioactive $\mathrm{TP}$ metabolites; the cytotoxicity of such metabolites is therefore increased by loss-of-function variants of NUDT15. In 2014, Yang et al. ${ }^{35}$ reported that a missense variant in exon 3 of the NUDT15 gene (p.Arg139Cys or R139C; single nucleotide polymorphism [SNP] ID: rs116855232) strongly correlates with TPinduced leukopenia among Korean patients with CD. Kakuta et al. ${ }^{36}$ confirmed this correlation in Japanese patients with CD. The high specificity and sensitivity (89.4\% and 93.2\%, respectively) of NUDT15 p.Arg139Cys indicate that this variant, rather than TPMT polymorphisms, may be an effective genetic marker for predicting TP-induced adverse events, at least in East Asian populations.

\section{Apheresis therapy for IBD}

Apheresis therapy is a treatment for IBD patients, that was developed in Japan. The mechanism of apheresis therapy is based on local immunomodulation achieved by removing leukocyte (granulocytes, monocytes, and activated-lymphocytes) from the peripheral blood with special columns. With no additive drugs, apheresis therapy appears to be a natural biologic therapy and may be an groundbreaking treatment method.

\section{1) Ulcerative colitis}

Regarding the efficacy and safety of apheresis, a metaanalysis that included nine randomized trials demonstrated that compared with CSs, granulocyte and monocyte adsorption apheresis (GMAA) is effective for inducing clinical remission in patients with active UC (OR, 2.23; 95\% CI, 1.38 to 3.60). ${ }^{37}$ In addition, the intensive apheresis ( $>2$ sessions per week) is more effective for inducing clinical remission than weekly apheresis (OR, 2.10; 95\% CI, 1.12 to 3.93). Notably, the rate of adverse events associated with apheresis was significantly lower than that associated with CSs (OR, 0.24; 95\% CI, 0.15 to 0.37). However, the disadvantage of apheresis is the lack of evidence concerning the efficacy of maintenance therapy. In Japan, a clinical trial is ongoing to determine whether apheresis is optimal as a maintenance therapy for UC patients. The role of apheresis in IBD treatment is still debated because most physicians have performed this therapy for patients with steroid-dependent or steroid-refractory UC. On the other hand, several reports have shown that the promising efficacy of apheresis in patients with steroid-naïve UC. The efficacy of apheresis in the induction of remission for patients with steroid-naïve active UC has not yet been established. A clinical trial investigating the efficacy of GMAA in patients with steroid-naïve active UC was recently finished and the results will be published soon.

\section{2) Crohn's disease}

Several reports have shown the efficacy of GMAA in patients with active $C D$. Based on data demonstrated that intensive GMAA is superior to weekly apheresis in the induction of remission for active UC, Yoshimura et al. ${ }^{38}$ performed an openlabel, prospective, randomized study to evaluate the clinical efficacy and safety of intensive GMAA in patients with active $\mathrm{CD}$. The results of the study demonstrated that the time to remission was significantly shorter in $\mathrm{CD}$ patients treated with intensive GMAA than in those treated with weekly GMAA, despite the absence of a significant difference in remission rates between CD patients treated with intensive GMAA and those treated with weekly GMAA. Fukuchi et al. ${ }^{39}$ studied the efficacy of GMAA combined with TPs in patients with early-diagnosed $\mathrm{CD}$. This study revealed that combination therapy with intensive GMAA and TPs administration rapidly induced full remission in patients with active early-diagnosed CD without serious adverse effects. Mucosal healing was observed in 50.0\% of enrolled patients. In this regard, this combination therapy might be a rational option for patients with early-diagnosed CD.

Many reports indicate that patients who respond to apheresis achieve a good long-term disease course by avoiding drugs including CSs in the early stage of their IBD. Therefore, in future therapeutic strategies, if we introduce apheresis at an early stage following the onset of IBD or before patients develop extensive mucosal damage and become refractory to medications, many IBD patients could respond to apheresis and avoid biologics.

\section{Calcineurin inhibitors}

Nuclear factor of activated T cells (NFAT) activation is required for inflammatory cytokines. To activate NFAT, calciumcalmodulin must activate calcineurin, which in turn dephosphorylates cytoplasmic NFAT, allowing it to move from the cytoplasm into the nucleus. Finally, nuclear NFAT binds to regulatory regions of genes that encode inflammatory cytokines (Fig. 6). Calcineurin inhibitors, such as cyclosporine A (CsA) and 
TAC, are available for the treatment of patients with refractory IBD.

\section{1) Cyclosporine $A$}

CsA binds to cyclophilin and TAC binds to the FK-binding protein. The inhibition of NFAT dephosphorylation by these complexes leads to the suppressed the transcription of inflammatory cytokine genes. ${ }^{40}$

\section{(1) Ulcerative colitis}

CsA has been used for the treatment of steroid-refractory severe UC. CsA has been used mainly as a rescue therapy in acute severe UC for over 2 decades. ${ }^{41}$ The first randomized placebocontrolled trial of CsA as a rescue therapy in acute severe steroid-refractory UC showed that nine out of 11 patients responded to CsA $4 \mathrm{mg} / \mathrm{kg}$ and avoided a colectomy, whereas no patient out of nine in the placebo group responded. ${ }^{42}$ Another randomized controlled trial (RCT) comparing $4 \mathrm{mg} / \mathrm{kg}$ with 2 $\mathrm{mg} / \mathrm{kg}$ IV CsA in 73 patients with severe UC showed that approximately $85 \%$ of the patients responded in each group, but the study included no placebo control. ${ }^{43}$ Thus, RCTs and several open studies have shown the efficacy of CsA in patients with severe UC who failed to respond to intravenous CSs. Overall, the short-term response rate after the induction of CsA was 64\% to $100 \%$, and the colectomy-free survival rate, an indicator of long-term prognosis, ranged from $14 \%$ to $55 \%$ within 3 to 7 years. ${ }^{40}$ However, there is limited evidence that CsA is more effective than the standard treatment for severe UC, and there is no evidence to support the long-term benefit of CsA. Generally, it is well known that UC patients who successfully achieve clinical remission with CsA should require adequate maintenance therapy with TPs.

\section{(2) Crohn's disease}

Stange et al. ${ }^{44}$ reported the result of a clinical trial evaluating 64 patients with active CD who were treated with CsA or placebo. There was no statistically significant difference between patients who received low-dose steroids and CsA $(5 \mathrm{mg} / \mathrm{kg}$ per day) and those who received low-dose steroids and placebo. In addition, there was no statistically significant benefit of CsA for preventing CD recurrence because over 70\% of both groups had relapsed at 1 year. Another RCT demonstrated that intravenous CsA was effective for patients with fistulizing $\mathrm{CD}$, although this trial only focused on the improvement of symptoms as an outcome. ${ }^{45}$ Overall, several reports indicated that the efficacy of intravenous or oral cyclosporine in patients with active $\mathrm{CD}$ or active UC was limited.

\section{2) TAC}

TAC is a macrolide antibiotic isolated from Streptomyces tsukubaensis that has immunomodulatory properties. TAC inhibits the formation of a complex between calcineurin and its $\mathrm{W}$ its cytoplasmic receptors cyclophilin and FK-binding protein 12 (FKBP-12), both of which regulate a calmodulin-dependent phosphatase, which interrupts signal transduction pathways in T cells. ${ }^{46,47}$ Notably, Yoshino et al. ${ }^{48}$ reported the dose-dependent inhibitory effect of TAC on proinflammatory cytokines (IL-12 and TNF- $\alpha$ ) from production by activated macrophages stimulated with lipopolysaccharide. Although its mode of action is similar to that of CsA, the immunosuppressive effect of TAC is 30 to 100 times greater in vitro and 10 to 20 times greater in vivo than that of CsA, and its intestinal absorption is more reliable, even in the presence of gastrointestinal disease. ${ }^{49}$

(1) Ulcerative colitis

TAC has changed the therapeutic strategy for moderately-

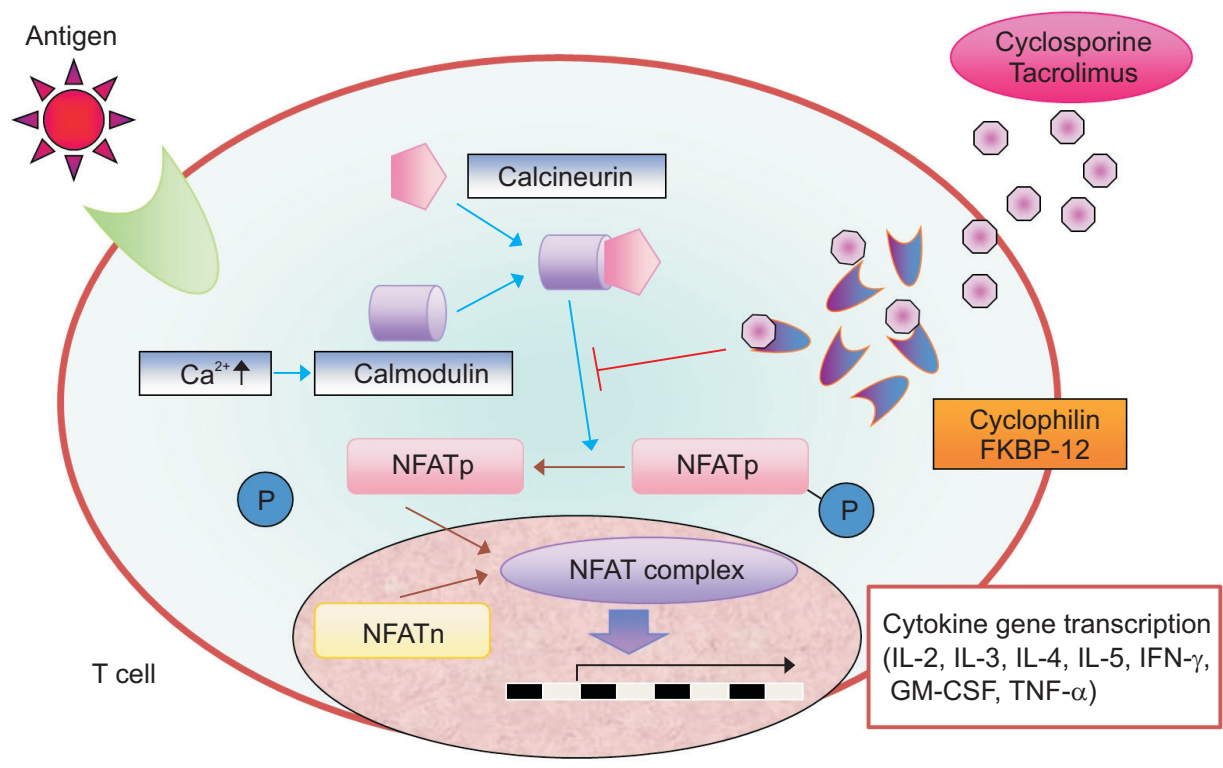

Fig. 6. Mechanism of action of tacrolimus.

NFAT, nuclear factor of activated T cells; NFATp, NFATc1 and NFATc2; NFATn, NFAT in the nucleus; $\mathrm{P}$, phosphoric acid; FKBP-12, FKbinding protein 12; IL, interleukin; IFN, interferon; GM-CSF, granulocyte macrophage colony-stimulating factors; TNF, tumor necrosis factor. Modified from Nakase $\mathrm{H}$. To master the optimal therapy for ulcerative colitis. Tokyo: Igakutokangosha Co. Ltd., 2018, with permission. ${ }^{2}$ 
severe active UC. Only one RCT comparing high target serum trough levels of TAC (10-15 ng/mL) and low trough levels of TAC (5-10 ng/mL) versus placebo for patients with steroidrefractory UC has been reported. ${ }^{50}$ The efficacy at 2 weeks after treatment demonstrated that significantly more patients responded in the high trough TAC group (68.4\%) than in the placebo group $(10.0 \%, \mathrm{p}<0.001)$. The low trough TAC groups showed a tendency toward response (38.1\%). A review from the Cochrane Central Register of Controlled Trials indicated that patients with UC in the high trough TAC group were significantly more likely to achieve clinical remission than patients in the other group. ${ }^{51}$ Therefore, rapidly increasing the trough level (10-15 $\mathrm{ng} / \mathrm{mL}$ ) in the blood to induce remission is important. A recent systematic review and meta-analysis that combined the data from these two trials and from observational studies demonstrated that the clinical response at 2 weeks was significantly stronger with TAC than with placebo. The colectomy-free rates at $1,3,6$, and 12 months were $0.86,0.84,0.78$, and 0.69, respectively. The long-term cumulative colectomy-free survival in UC patients with TAC has been reported to be 57\% at 44 months, although this study included a very heterogeneous population. $^{52}$ Several uncontrolled series have shown that TAC is effective for the treatment of patients with UC who are intolerant or refractory to conventional therapies. ${ }^{52-55}$ Like patients treated with CsA, steroid-refractory UC patients who achieve remission with TAC treatment would require immunomodulators to maintain remission and prevent colectomy. Recently, Schmidt et al. ${ }^{56}$ reported the effect of purine analogs (PAs) on the long-term clinical outcomes of 156 moderately to severely active steroid-refractory UC patients treated with TAC. The Kaplan-Meier curve for colectomy-free survival after month 3 showed a benefit in the PA group. The time to colectomy was 2 years (median; range, 0.7 to 5.8 ) in the PA group and 0.8 years (range, 0.3 to 4.7 years) in the group that was not treated with PAs ( $p=0.02$ ). The time to relapse was 1.2 years (median; range 0.3 to 6.2 years) in patients with PA treatment and 0.5 years (range, 0.3 to 3.9 years) in those without PA treatment ( $\mathrm{p}=0.05)$. These data strongly suggested that the addition of TP therapy significantly correlated with colectomy avoidance and remission rates.

\section{(2) Crohn's disease}

Yoshino et $a .^{48}$ reported that TAC directly suppressed the LPS-induced activation of both NF- $\mathrm{KB}$ and MAPK in macrophages and induced the apoptosis of macrophages through the activation of caspase- 3 and caspase-9. In addition, IL-12/IL23p40, IL-6, and TNF- $\alpha$ production by activated macrophages were inhibited by TAC in a dose-dependent manner. It is well known that the IL-12/IL-23p40 pathway is deeply involved in the pathogenesis of $\mathrm{CD} .^{57}$ Taken together, these basic data suggest the efficacy of TAC therapy in patients with active CD. In real clinical practice, the efficacy of TAC in the management of
$\mathrm{CD}$ is under debate because of the lack of randomized control trials. McSharry et al.$^{58}$ systematically reviewed the efficacy and safety of TAC in treating CD. The author's assessed case series, cohort studies, and RCTs that incorporated oral, intravenous, or topical TAC therapy. In patients with luminal $\mathrm{CD}$, the crude pooled remission rate for TAC was $44.3 \%$ (range, 7\% to 69\%), and the crude pooled response rate was 37.1\% (range, 14\% to 57\%). Additionally, in patients with CD who had the perianal disease and were treated with systemic TAC, the crude pooled remission rate was $28.6 \%$ (range, $0 \%$ to $64 \%$ ), and the crude pooled response rate was $38.8 \%$ (range, $0 \%$ to $57 \%$ ). Data from two studies of topical TAC showed that 35.7\% of patients achieved remission and 28.6\% achieved a partial response. Gerich et al. ${ }^{59}$ showed a retrospective study of oral TAC for severe CD refractory to anti-TNF agents. Twenty-four patients with CD were treated with oral TAC for a median of 4 months. The response and steroid-free remission rates were 67\% and $21 \%$, respectively. Approximately $42 \%$ of patients were able to stop steroids, and 54\% of patients ultimately required surgery within a median of 10 months after starting TAC. Of note, patients with mean trough TAC levels of 10 to $15 \mathrm{ng} / \mathrm{mL}$ had the highest rates of response (86\%) and remission (57\%). This study suggested that oral TAC might be promising for some patients with severe CD that is refractory to anti-TNF- $\alpha$ therapies. Clinical series and meta-analyses have suggested that both CsA and TAC are promising for patients with steroid-refractory severe UC and active CD refractory to TNF inhibitors. However, maintaining the optimal trough level in the blood is essential for the rapid induction of remission when using CsA and TAC. Moreover, in the case of severe UC, physicians should avoid missing the optimal timing for surgical treatment.

\section{Biologics}

We are currently in the era of biologics for IBD treatment. In Japan, infliximab, adalimumab and golimumab, and ustekinumab are now available. The emergence of biologics has drastically changed the natural history of CD and UC. Additionally, treatment with TNF inhibitors has changed several aspects of treatment, including therapeutic concepts, disease concepts, and therapeutic goals.

\section{1) Change in the evaluation of disease activity}

The previous clinical trial evaluated the efficacy of several new drugs in IBD patients by using a clinical symptoms score, such as the CD activity index or the clinical activity index. Currently, disease activity is evaluated objectively based on endoscopic findings, calprotectin, and magnetic resonance imaging. Among these approaches, the relevance of the endoscopic activity of UC has been translated into the new concept of "mucosal healing" as a therapeutic goal because accumulating evidence indicates the favorable prognostic value of a healed mucosa in the clinical outcome of IBD. In particular, mucosal healing as- 
sessed by endoscopy seems to be the gold standard for evaluating UC activity. Moreover, the concept of "deep remission" has also emerged. With these changes in the evaluation of disease activity, the "treat to target" strategy, which uses objective clinical and biochemical outcome measures to assist clinicians in making decisions related to therapy modification, has spread in the IBD field.

\section{2) The emergence of the concept of "top-down" in IBD treatment}

The term "top-down" in the context of IBD therapy, particularly for CD patients, means that biologicals and immunosuppressants are applied immediately after the diagnosis of CD as a first-line therapy. A landmark paper on top-down therapy by D'Haens et al..$^{0}$ demonstrated that early combined immunosuppression was superior to conventional management. Later, REACT study suggested that early combined immunosuppression was not more effective than conventional management for controlling CD symptoms; however, this approach could avoid complications related to $\mathrm{CD} .^{61}$ The rationale for such an approach comes from rheumatology where an early intervention with biologicals is thought to be "disease-modifying," for example, such an approach prevents the progressive destruction of joints. However, if a top-down therapeutic approach is recommended for all patients diagnosed with $\mathrm{CD}$, a significant number of patients will be overtreated.

\section{3) TNF- inhibitors}

TNF is a prototypic member of a large family of cytokines that play important roles in the regulation of the innate and adaptive immune system. TNF itself is a key player in the initiation and orchestration of inflammation and immunity and is a potent proinflammatory cytokine, which is predominantly produced by activated immune cells in response to infections and during tissue repair (Fig. 7). However, the dysregulation of TNF expression and signaling can cause chronic inflammation, which could result in the development of autoimmune diseases and tissue damage. In fact, elevated TNF levels have been associated with disease activity in several diseases such as IBD. In this context, treatment with TNF inhibitors has been successfully performed.

\section{4) Clinical trial data for several TNF inhibitors}

TNF inhibitors, such as infliximab and adalimumab, have proven to be effective for CD and UC in various controlled trials. ${ }^{62-66}$ On the other hand, we encountered an important clinical issue regarding how to treat primary nonresponders and second nonresponders (who lose clinical remission over time) to TNF inhibitors. Several investigators have shown that approximately one-third of $\mathrm{CD}$ patients suffer primary response failure. In the case of primary nonresponder, we must consider that complications, such as severe stricture, intestinal fistula, and abdominal abscess, influence the therapeutic efficacy of TNF inhibitors, and the patient's condition affects the pharmacokinetics of TNF inhibitors and the involvement of other inflammatory pathways beyond TNF in pathogenesis. In the case of secondary nonresponders, several clinical trials showed that $40 \%$ to $50 \%$ of patients with CD who responded to an anti-TNF agent lost the response within 6 to 12 months. The development of antibodies against anti-TNF drugs and the associated low serum trough concentrations of drugs have been implicated as predisposing factors to secondary failure in IBD patients treated with TNF inhibitors. To overcome this issue, we should improve unsatisfactory patient compliance in connection with the selfadministration of drugs (if it exists), and intensify therapy using increased dosages and/or shortened intervals of administration

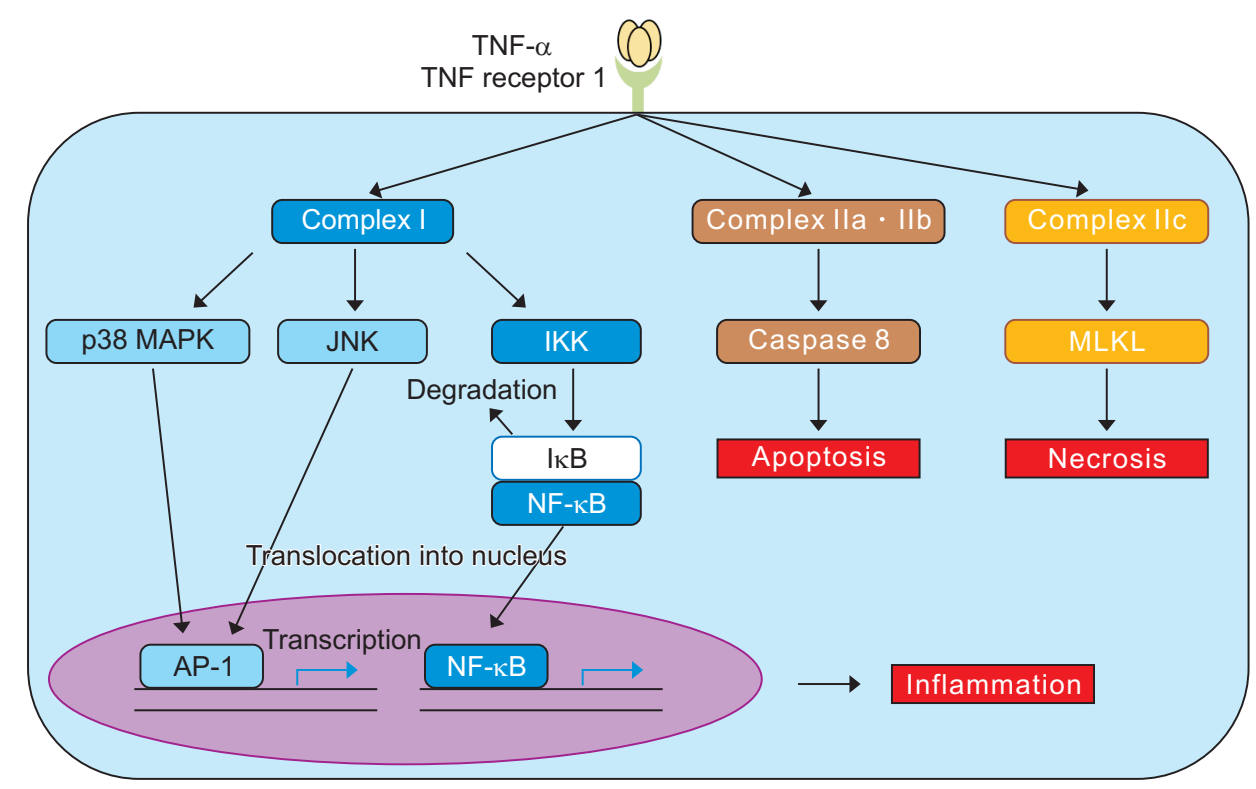

Fig. 7. Mechanism of action of tumor necrosis factor (TNF) inhibitors. MAPK, mitogen-activated protein kinase; JNK, c-Jun $\mathrm{N}$ terminal kinase; IKK, inhibitor of kappa B kinase; $I_{\kappa} B$, nuclear factor of kappa light polypeptide gene enhancer in $\mathrm{B}$-cells inhibitor; NF- $\mathrm{BB}$, nuclear facor- $\kappa \mathrm{B}$; AP-1, activator protein1; MLKL, mixed lineage kinase domain-like protein. Modified from Nakase H. To master the optimal therapy for ulcerative colitis. Tokyo: Igakutokangosha Co., Ltd., 2018, with permission. ${ }^{2}$ 
of the same TNF- $\alpha$ inhibitor concomitantly with immunomodulators.

Recently, golimumab (a fully human monoclonal IgG1 antibody developed by Janssen Biotech, Inc.) was approved for moderately to severely active UC patients who have had an inadequate response to conventional therapy including CSs and 6-MP and AZA, or who are intolerant to or have medical contraindications for such therapies. ${ }^{67}$ The 4 -week dosing interval for golimumab may be explained by the fact that in vitro and in vivo affinity of golimumab for soluble human TNF- $\alpha$ is significantly higher than that of adalimumab. However, we must remember that the role of golimumab in treating acute severe colitis remains to be determined. In clinical practice, intravenous infliximab appears to be the most effective and suitable therapy because of its rapid action.

\section{5) Anti-IL12/23 antibody (ustekinumab)}

IL-12 and IL-23 are produced by antigen-presenting cells (APCs) during intestinal inflammation. ${ }^{68}$ Dendritic cells and macrophages showed augmented production of IL-12 (which is composed of the p35 and p40 subunits) and IL-23 (which is composed of the IL-12 p40 subunit and a p19 subunit). IL-12 signals through STAT4 and the central role of this transcription factor in skewing naïve Th cells toward the Th1 phenotype has been well documented. IL-23 triggers the heterodimerization of IL-12R $\beta 1$ and IL-23R. Subsequently, signal transduction pathways, including Tyk2 and JAK2 /STAT4 and 3, are activated. IL23 plays an important role in expanding and maintaining the Th-17 cell population. Preclinical studies have implicated IL-12 and IL-23 in the pathophysiology of $\mathrm{CD}^{69}$ In addition, genome- wide association studies have shown an association between the IL-12/IL-23 pathway and CD. ${ }^{70}$ Ustekinumab, a fully human IgG1k monoclonal antibody, blocks the biologic activity of IL12 and IL-23 through their common p40 subunit by inhibiting receptors for these two cytokines on T cells, natural killer cells, and APCs (Fig. 8). Ustekinumab demonstrated therapeutic efficacy in induction and maintenance therapy in patients with moderately and severely active CD in the UNITI trials. ${ }^{71} \mathrm{Al}-$ though the use of ustekinumab for up to 5 years is safe and has
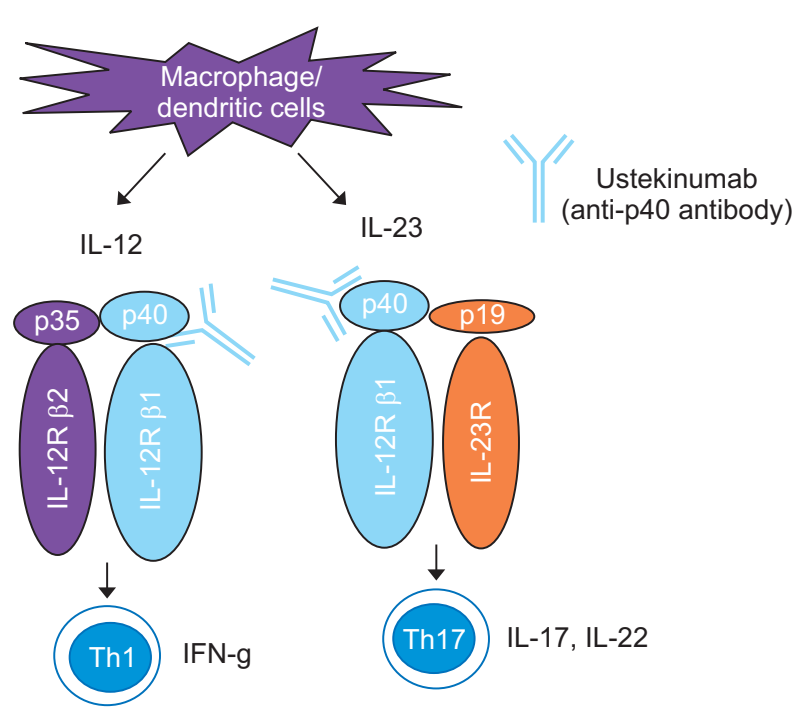

Fig. 8. Mechanism of action of ustekinumab.

IL, interleukin; IFN, interferon. Modified from Nakase H. To master the optimal therapy for ulcerative colitis. Tokyo: Igakutokangosha Co., Ltd., 2018, with permission. ${ }^{2}$

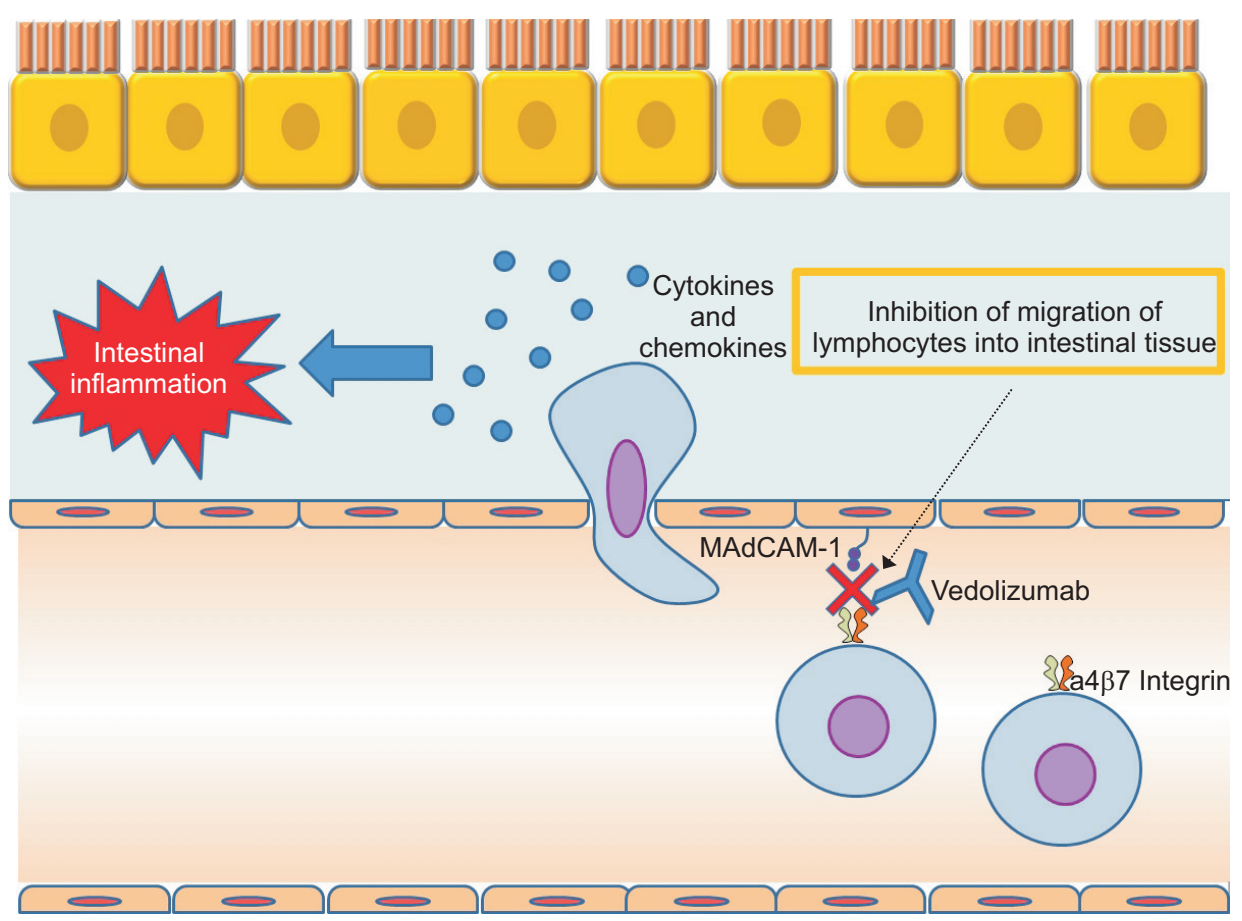

Fig. 9. Mechanism of vedolizumab. Modified from Nakase H. To master the optimal therapy for ulcerative colitis. Tokyo: Igakutokangosha Co., Ltd., 2018, with permission. ${ }^{2}$ 


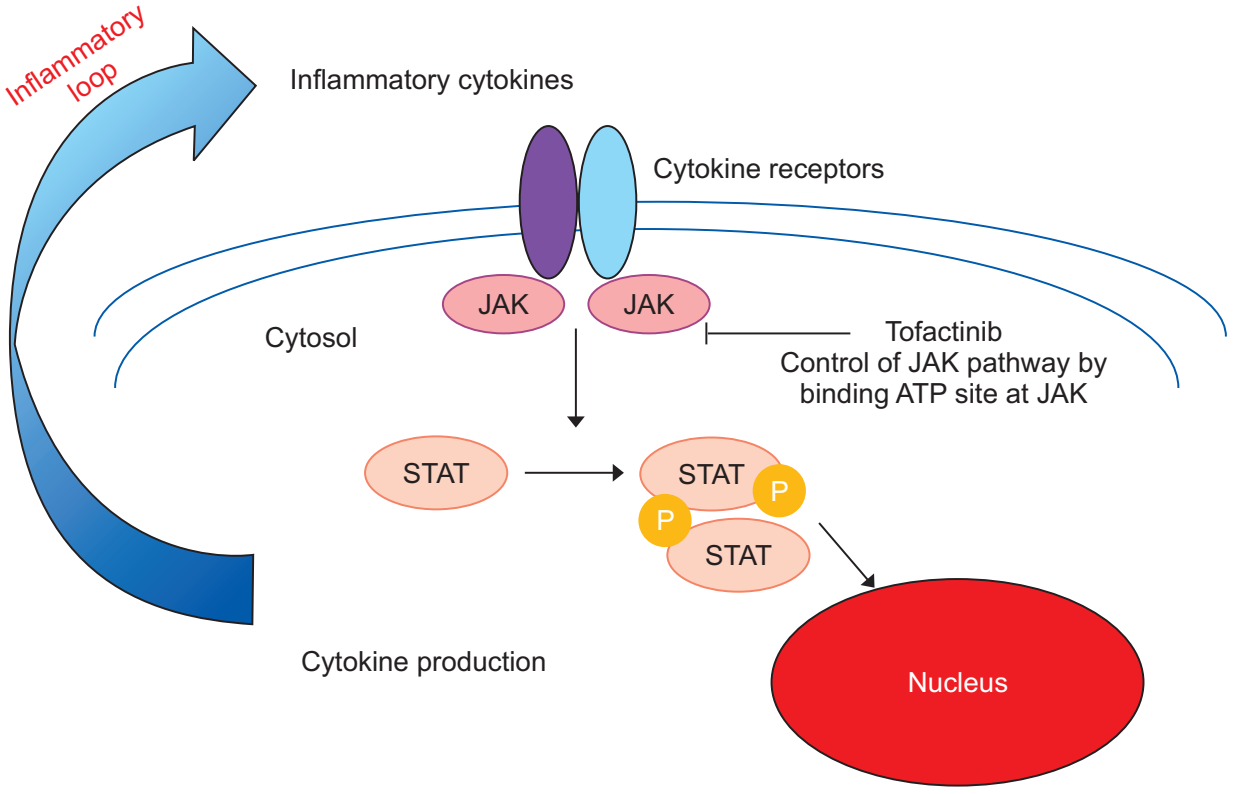

Fig. 10. Mechanism of Janus kinase (JAK) inhibitors.

STAT, signal transducer and activator of transcription; $\mathrm{P}$, phosphoric acid. Modified from Nakase H. To master the optimal therapy for ulcerative colitis. Tokyo: Igakutokangosha Co., Ltd., 2018, with permission. ${ }^{2}$ no increased risk of malignancy based on experience in plaque psoriasis and psoriatic arthritis, more clinical data regarding the long-term safety of this drugs in CD patients is needed.

\section{6) Anti-adhesion molecules}

Vedolizumab, a mAb that targets $\alpha 4 \beta 7$ integrins, resulting in a gut-selective mechanism of action, has emerged for the induction of remission in moderately active UC patients (Fig. 9). ${ }^{72}$ Vedolizumab is considered to have a lower risk of progressive multifocal leukoencephalopathy than natalizumab, which binds the $\alpha 4 \beta 1$ integrin, that is involved in brain lymphocyte trafficking. ${ }^{73}$ This biologic is expected to be a second-line biologic agent after anti-TNF agents. In clinical practice, physicians might be aware of the potentially slower onset of action of vedolizumab. Therefore, the combination with CSs or calcineurin inhibitors or TNF inhibitors should be considered during the induction period. ${ }^{74,75}$ In this regard, TNF inhibitors or ustekinumab may be more favorable as a first-line therapy in patients with severe active $\mathrm{CD}$.

\section{JAK inhibitors}

The JAK enzymes, which are named after the two-faced Roman god Janus, play important roles in the signaling pathways of several cytokines through their receptors. ${ }^{76}$ Different combinations of JAK1, 2, 3 and tyrosine kinase (TYK) 2 are involved in the signaling of inflammatory cytokines. For example, the JAK1 and JAK3 are tyrosine kinases that mediate signal transduction activity involving the common gamma chain of the surface receptors for multiple cytokines, including IL-2, IL-4, IL7, IL-9, IL-15, and IL-21. The JAK inhibitor tofacitinib, which selectively inhibits JAK1 and JAK3, has been used for the treatment of rheumatoid arthritis and psoriasis (Fig. 10). Regarding the efficacy of tofacitinib in patients with moderate to severe
UC, the results of two large phase III trials (Octave 1 and 2) confirmed the efficacy of tofacitinib in inducing and maintaining remission. ${ }^{77}$

Tofacitinib appears to be safe. However, the long-term safety of this agent in the treatment of UC remains unclear. Additionally, we must deliberately administer tofacitinib to elderly patients with UC because clinical trial data showed an increasing risk of Herpes zoster in patients with UC who were greater than 65-year-old.

\section{Future directions}

What is the next step? To move toward a "treat to target" strategy, objective assessment of inflammation is now critical. Therefore, endoscopic, histological and radiological assessment tools, as well as the use of biomarkers (calprotectin and fecal immunochemical test) and quality of life evaluation, should be taken into consideration as domains of disease activity assessment in patients with IBD.

The management of IBD has been transformed over the past two decades by the emergence of several biologics. Recently, new drugs have been approved, and these drugs target leukocyte-trafficking molecules (vedolizumab) or other inflammatory cytokines (ustekinumab). Brand-new treatments are currently being developed for IBD and represent promising targets as they involve other mechanisms of action (JAK molecules, sphingosine-1-phosphate receptor modulators, etc.). Beyond TNF antagonist agents, these alternative drugs might be needed for early-stage treatment in patients with aggressive IBD or patients whose disease is resistant to conventional therapy. Thus, the current clinical issue is to decide the optimal roles of several new drugs in IBD treatment, and more clinical data are required to confirm the long-term safety of each drug. Fecal microbiota transplantation and treatments with nucleotides and mesenchy- 
mal stem cells, are expected to become available in the future; however, the clinical application of these treatments is premature. Personalized medicine is the next step for IBD patients. In the future, finding patients who are at high risk of progression or complications and better characterizing patients who respond preferentially to specific therapies is essential for the optimal management of patients with IBD.

Finally, I would like to say "Be familiar with the mechanisms of action of the drug and know your IBD patients, and you make them happy."

\section{CONFLICTS OF INTEREST}

No potential conflict of interest relevant to this article was reported.

\section{ACKNOWLEDGEMENTS}

This work was supported by Health and Labour Sciences Research Grants for research on intractable diseases from the Ministry of Health, Labour and Welfare of Japan (Investigation and Research for intractable Inflammatory Bowel Disease) (to H.N.), and Japan Society for the Promotion of Science (JSPS) Grants-in-Aid for Scientific Research (KAKENHI) Grant Number JP18H02799 (to H.N.).

\section{REFERENCES}

1. Nakase H. How to select and use IBD drugs. Tokyo: Yodosha Co., Ltd., 2015.

2. Nakase H. To master the optimal therapy for ulcerative colitis. Tokyo: Igakutokangosha Co., Ltd., 2018.

3. Stolfi C, De Simone V, Pallone F, Monteleone G. Mechanisms of action of non-steroidal anti-inflammatory drugs (NSAIDs) and mesalazine in the chemoprevention of colorectal cancer. Int J Mol Sci 2013;14:17972-17985.

4. Oh-Oka K, Kojima Y, Uchida K, et al. Induction of colonic regulatory $\mathrm{T}$ cells by mesalamine by activating the aryl hydrocarbon receptor. Cell Mol Gastroenterol Hepatol 2017;4:135-151.

5. Marshall JK, Irvine EJ. Rectal aminosalicylate therapy for distal ulcerative colitis: a meta-analysis. Aliment Pharmacol Ther 1995;9:293-300.

6. Nakase H, Keum B, Ye BD, Park SJ, Koo HS, Eun CS. Treatment of inflammatory bowel disease in Asia: the results of a multinational web-based survey in the 2(nd) Asian Organization of Crohn's and Colitis (AOCC) meeting in Seoul. Intest Res 2016;14:231-239.

7. Sutherland L, Macdonald JK. Oral 5-aminosalicylic acid for induction of remission in ulcerative colitis. Cochrane Database Syst Rev 2006;(2):CD000543.

8. Ogata H, Aoyama N, Mizushima S, Hagino A, Hibi T. Comparison of efficacy of multimatrix mesalazine $4.8 \mathrm{~g} /$ day once-daily with other high-dose mesalazine in active ulcerative colitis: a random- ized, double-blind study. Intest Res 2017;15:368-379.

9. Sutherland L, Macdonald JK. Oral 5-aminosalicylic acid for maintenance of remission in ulcerative colitis. Cochrane Database Syst Rev 2006;(2):CD000544.

10. Flourié B, Hagège $H$, Tucat G, et al. Randomised clinical trial: once- vs. twice-daily prolonged-release mesalazine for active ulcerative colitis. Aliment Pharmacol Ther 2013;37:767-775.

11. Kruis W, Jonaitis L, Pokrotnieks J, et al. Randomised clinical trial: a comparative dose-finding study of three arms of dual release mesalazine for maintaining remission in ulcerative colitis. Aliment Pharmacol Ther 2011;33:313-322.

12. Lichtenstein GR, Hanauer SB, Sandborn WJ; Practice Parameters Committee of American College of Gastroenterology. Management of Crohn's disease in adults. Am J Gastroenterol 2009;104:465483.

13. Coward S, Kuenzig ME, Hazlewood G, et al. Comparative effectiveness of mesalamine, sulfasalazine, corticosteroids, and budesonide for the induction of remission in Crohn's disease: a Bayesian network meta-analysis. Inflamm Bowel Dis 2017;23:461-472.

14. Mowat C, Cole A, Windsor A, et al. Guidelines for the management of inflammatory bowel disease in adults. Gut 2011;60:571607.

15. Nenci A, Becker C, Wullaert A, et al. Epithelial NEMO links innate immunity to chronic intestinal inflammation. Nature 2007;446:557-561.

16. Inoue S, Nakase H, Matsuura M, et al. The effect of proteasome inhibitor MG132 on experimental inflammatory bowel disease. Clin Exp Immunol 2009;156:172-182.

17. Creed TJ, Probert CS. Review article: steroid resistance in inflammatory bowel disease - mechanisms and therapeutic strategies. Aliment Pharmacol Ther 2007;25:111-122.

18. Ford AC, Bernstein CN, Khan KJ, et al. Glucocorticosteroid therapy in inflammatory bowel disease: systematic review and metaanalysis. Am J Gastroenterol 2011;106:590-599.

19. Hart AL, Ng SC. Review article: the optimal medical management of acute severe ulcerative colitis. Aliment Pharmacol Ther 2010;32:615-627.

20. Baron JH, Connell AM, Kanaghinis TG, Lennard-Jones JE, Jones AF. Out-patient treatment of ulcerative colitis: comparison between three doses of oral prednisone. Br Med J 1962;2:441-443.

21. Dignass A, Van Assche G, Lindsay J0, et al. The second European evidence-based consensus on the diagnosis and management of Crohn's disease: current management. J Crohns Colitis 2010;4:2862.

22. Lichtenstein GR, Abreu MT, Cohen R, Tremaine W; American Gastroenterological Association. American Gastroenterological Association Institute technical review on corticosteroids, immunomodulators, and infliximab in inflammatory bowel disease. Gastroenterology 2006;130:940-987.

23. Turner D, Walsh CM, Steinhart AH, Griffiths AM. Response to corticosteroids in severe ulcerative colitis: a systematic review of the literature and a meta-regression. Clin Gastroenterol Hepatol 
2007;5:103-110.

24. Shin JY, Wey M, Umutesi HG, Sun X, Simecka J, Heo J. Thiopurine prodrugs mediate immunosuppressive effects by interfering with Rac1 protein function. J Biol Chem 2016;291:13699-13714.

25. Gisbert JP, Linares PM, McNicholl AG, Maté J, Gomollón F. Metaanalysis: the efficacy of azathioprine and mercaptopurine in ulcerative colitis. Aliment Pharmacol Ther 2009;30:126-137.

26. Yamada S, Yoshino T, Matsuura M, et al. Efficacy and safety of long-term thiopurine maintenance treatment in Japanese patients with ulcerative colitis. Intest Res 2015;13:250-258.

27. Pearson DC, May GR, Fick G, Sutherland LR. Azathioprine for maintaining remission of Crohn's disease. Cochrane Database Syst Rev 2000;(2):CD000067.

28. Lee KM, Kim YS, Seo GS, Kim TO, Yang SK; IBD Study Group of the Korean Association for the Study of Intestinal Diseases. Use of thiopurines in inflammatory bowel disease: a consensus statement by the Korean Association for the Study of Intestinal Diseases (KASID). Intest Res 2015;13:193-207.

29. Pinto AL, Chebli LA, Ribeiro MS, et al. Azathioprine therapy in steroid-dependent patients with Crohn disease: results of a 10-year longitudinal follow-up study. Med Sci Monit 2009;15:PI19-PI26.

30. Chebli LA, Chaves LD, Pimentel FF, et al. Azathioprine maintains long-term steroid-free remission through 3 years in patients with steroid-dependent ulcerative colitis. Inflamm Bowel Dis 2010;16:613-619.

31. Takagi Y, Setoyama D, Ito R, Kamiya H, Yamagata Y, Sekiguchi M. Human MTH3 (NUDT18) protein hydrolyzes oxidized forms of guanosine and deoxyguanosine diphosphates: comparison with MTH1 and MTH2. J Biol Chem 2012;287:21541-21549.

32. Carter M, Jemth AS, Hagenkort A, et al. Crystal structure, biochemical and cellular activities demonstrate separate functions of MTH1 and MTH2. Nat Commun 2015;6:7871.

33. Valerie NC, Hagenkort A, Page BD, et al. NUDT15 hydrolyzes 6-Thio-DeoxyGTP to mediate the anticancer efficacy of 6-thioguanine. Cancer Res 2016;76:5501-5511.

34. Moriyama T, Nishii R, Perez-Andreu V, et al. NUDT15 polymorphisms alter thiopurine metabolism and hematopoietic toxicity. Nat Genet 2016;48:367-373.

35. Yang SK, Hong M, Baek J, et al. A common missense variant in NUDT15 confers susceptibility to thiopurine-induced leukopenia. Nat Genet 2014;46:1017-1020.

36. Kakuta Y, Naito T, Onodera M, et al. NUDT15 R139C causes thiopurine-induced early severe hair loss and leukopenia in Japanese patients with IBD. Pharmacogenomics J 2016;16:280-285.

37. Yoshino T, Nakase H, Minami N, et al. Efficacy and safety of granulocyte and monocyte adsorption apheresis for ulcerative colitis: a meta-analysis. Dig Liver Dis 2014;46:219-226.

38. Yoshimura N, Yokoyama Y, Matsuoka K, et al. An open-label prospective randomized multicenter study of intensive versus weekly granulocyte and monocyte apheresis in active Crohn's disease. BMC Gastroenterol 2015;15:163.

39. Fukuchi T, Nakase H, Ubukata S, et al. Therapeutic effect of inten- sive granulocyte and monocyte adsorption apheresis combined with thiopurines for steroid- and biologics-naïve Japanese patients with early-diagnosed Crohn's disease. BMC Gastroenterol 2014;13:124.

40. Naganuma M, Fujii T, Watanabe M. The use of traditional and newer calcineurin inhibitors in inflammatory bowel disease. $\mathrm{J}$ Gastroenterol 2011;46:129-137.

41. Lichtiger S. Treatment of choice for acute severe steroid-refractory ulcerative colitis is cyclosporine. Inflamm Bowel Dis 2009;15:141142.

42. Lichtiger S, Present DH, Kornbluth A, et al. Cyclosporine in severe ulcerative colitis refractory to steroid therapy. N Engl J Med 1994;330:1841-1845.

43. Van Assche G, D’Haens G, Noman M, et al. Randomized, double-blind comparison of $4 \mathrm{mg} / \mathrm{kg}$ versus $2 \mathrm{mg} / \mathrm{kg}$ intravenous cyclosporine in severe ulcerative colitis. Gastroenterology 2003;125:1025-1031.

44. Stange EF, Modigliani R, Peña AS, Wood AJ, Feutren G, Smith PR. European trial of cyclosporine in chronic active Crohn's disease: a 12-month study. The European Study Group. Gastroenterology 1995;109:774-782.

45. Brynskov J, Freund L, Rasmussen SN, et al. A placebo-controlled, double-blind, randomized trial of cyclosporine therapy in active chronic Crohn's disease. N Engl J Med 1989;321:845-850.

46. Rusnak F, Mertz P. Calcineurin: form and function. Physiol Rev 2000;80:1483-1521.

47. Wong SH. Therapeutic drug monitoring for immunosuppressants. Clin Chim Acta 2001;313:241-253.

48. Yoshino T, Nakase H, Honzawa Y, et al. Immunosuppressive effects of tacrolimus on macrophages ameliorate experimental colitis. Inflamm Bowel Dis 2010;16:2022-2033.

49. Kino T, Hatanaka H, Miyata S, et al. FK-506, a novel immunosuppressant isolated from a Streptomyces. II. Immunosuppressive effect of FK-506 in vitro. J Antibiot (Tokyo) 1987;40:1256-1265.

50. Ogata H, Matsui T, Nakamura M, et al. A randomised dose finding study of oral tacrolimus (FK506) therapy in refractory ulcerative colitis. Gut 2006;55:1255-1262.

51. Baumgart DC, Macdonald JK, Feagan B. Tacrolimus (FK506) for induction of remission in refractory ulcerative colitis. Cochrane Database Syst Rev 2008;(3):CD007216.

52. Yamamoto S, Nakase H, Mikami S, et al. Long-term effect of tacrolimus therapy in patients with refractory ulcerative colitis. Aliment Pharmacol Ther 2008;28:589-597.

53. Fellermann K, Tanko Z, Herrlinger KR, et al. Response of refractory colitis to intravenous or oral tacrolimus (FK506). Inflamm Bowel Dis 2002;8:317-324.

54. Högenauer C, Wenzl HH, Hinterleitner TA, Petritsch W. Effect of oral tacrolimus (FK 506) on steroid-refractory moderate/severe ulcerative colitis. Aliment Pharmacol Ther 2003;18:415-423.

55. Baumgart DC, Pintoffl JP, Sturm A, Wiedenmann B, Dignass AU. Tacrolimus is safe and effective in patients with severe steroidrefractory or steroid-dependent inflammatory bowel disease: a 
long-term follow-up. Am J Gastroenterol 2006;101:1048-1056.

56. Schmidt KJ, Müller N, Dignass A, et al. Long-term outcomes in steroid-refractory ulcerative colitis treated with tacrolimus alone or in combination with purine analogues. J Crohns Colitis 2016;10:31-37.

57. Niederreiter L, Adolph TE, Kaser A. Anti-IL-12/23 in Crohn's disease: bench and bedside. Curr Drug Targets 2013;14:1379-1384.

58. McSharry K, Dalzell AM, Leiper K, El-Matary W. Systematic review: the role of tacrolimus in the management of Crohn's disease. Aliment Pharmacol Ther 2011;34:1282-1294.

59. Gerich ME, Pardi DS, Bruining DH, Kammer PP, Becker BD, Tremaine WT. Tacrolimus salvage in anti-tumor necrosis factor antibody treatment-refractory Crohn's disease. Inflamm Bowel Dis 2013;19:1107-1111.

60. D'Haens G, Baert F, van Assche G, et al. Early combined immunosuppression or conventional management in patients with newly diagnosed Crohn's disease: an open randomized trial. Lancet 2008;371:660-667.

61. Khanna R, Bressler B, Levesque BG, et al. Early combined immunosuppression for the management of Crohn's disease (REACT): a cluster randomised controlled trial. Lancet 2015;386:1825-1834.

62. Present DH, Rutgeerts P, Targan S, et al. Infliximab for the treatment of fistulas in patients with Crohn's disease. N Engl J Med 1999;340:1398-1405.

63. Colombel JF, Sandborn WJ, Reinisch W, et al. Infliximab, azathioprine, or combination therapy for Crohn's disease. N Engl J Med 2010;362:1383-1395.

64. Hanauer SB, Sandborn WJ, Rutgeerts P, et al. Human anti-tumor necrosis factor monoclonal antibody (adalimumab) in Crohn's disease: the CLASSIC-I trial. Gastroenterology 2006;130:323-333.

65. Rutgeerts P, Sandborn WJ, Feagan BG, et al. Infliximab for induction and maintenance therapy for ulcerative colitis. N Engl J Med 2005;353:2462-2476.

66. Sandborn WJ, Rutgeerts P, Feagan BG, et al. Colectomy rate comparison after treatment of ulcerative colitis with placebo or inflix- imab. Gastroenterology 2009;137:1250-1260.

67. Limdi JK. Golimumab for ulcerative colitis: adding perspective to the pursuit. Frontline Gastroenterol 2018;9:232-233.

68. Neurath MF. Cytokines in inflammatory bowel disease. Nat Rev Immunol 2014;14:329-342.

69. Neurath MF. IL-23: a master regulator in Crohn disease. Nat Med 2007;13:26-28.

70. Wang K, Zhang H, Kugathasan S, et al. Diverse genome-wide association studies associate the IL12/IL23 pathway with Crohn disease. Am J Hum Genet 2009;84:399-405.

71. Feagan BG, Sandborn WJ, Gasink C, et al. Ustekinumab as Induction and maintenance therapy for Crohn's disease. N Engl J Med 2016;375:1946-1960.

72. Feagan BG, Rutgeerts P, Sands BE, et al. Vedolizumab as induction and maintenance therapy for ulcerative colitis. N Engl J Med 2013;369:699-710.

73. Soler D, Chapman T, Yang LL, Wyant T, Egan R, Fedyk ER. The binding specificity and selective antagonism of vedolizumab, an anti-alpha4beta7 integrin therapeutic antibody in development for inflammatory bowel diseases. J Pharmacol Exp Ther 2009;330:864-875.

74. Christensen B, Gibson PR, Micic D, et al. Safety and efficacy of combination treatment with calcineurin inhibitors and vedolizumab in patients with refractory inflammatory bowel disease. Clin Gastroenterol Hepatol 2019;17:486-493.

75. Buer LCT, Høivik ML, Warren DJ, Medhus AW, Moum BA. Combining anti-TNF-alpha and vedolizumab in the treatment of inflammatory bowel disease: a case series. Inflamm Bowel Dis 2018;24:997-1004.

76. Verstockt B, Ferrante M, Vermeire S, Van Assche G. New treatment options for inflammatory bowel diseases. J Gastroenterol 2018;53:585-590.

77. Sandborn WJ, Su C, Sands BE, et al. Tofacitinib as induction and maintenance therapy for ulcerative colitis. N Engl J Med 2017;376:1723-1736. 